\title{
Pressurized IntraPeritoneal Aerosol Chemotherapy - Practical aspects
}

\author{
M. Hübner ${ }^{\text {a } *, 1}$, F. Grass ${ }^{\text {a,1 }}$, H. Teixeira-Farinha ${ }^{\text {a }}$, B. Pache ${ }^{\text {a }}$, \\ P. Mathevet ${ }^{b}$, N. Demartines ${ }^{a}$ \\ ${ }^{a}$ Department of Visceral Surgery, University Hospital of Lausanne (CHUV), 1011 Lausanne, Switzerland \\ ${ }^{\mathrm{b}}$ Department of Gynecology, University Hospital of Lausanne (CHUV), 1011 Lausanne, Switzerland
}

Accepted 29 March 2017

Available online 8 April 2017

\begin{abstract}
Introduction: Pressurized Intraperitoneal Aerosol Chemotherapy (PIPAC) has been introduced as novel treatment for peritoneal carcinomatosis. Only proper patient selection, stringent safety protocol and careful surgery allow for a secure procedure. We hereby report the essentials for safe implementation.

Methods: All consecutive procedures within 20 months after PIPAC implementation were analyzed with regards to practical and surgical aspects. Special emphasis was laid on modifications of technique and safety measures during the implementation process with systematic use of a dedicated checklist. Further, surgical difficulty was documented by use of a visual analogue scale (VAS).

Results: 127 PIPAC procedures were performed in 58 patients from January 2015 until October 2016. 81\% of patients had at least one previous laparotomy. Median operation time was $91 \mathrm{~min}$ (87-103) for the first 20 cases, 93 min (IQR 88-107) for PIPAC21-50, and $103 \mathrm{~min}$ (IQR 91-121) for the following 77 procedures. Primary and secondary non-access occurred in 3 patients (2\%), all of them having prior hyperthermic intraperitoneal chemotherapy (HIPEC). Using open Hasson technique, one single bowel lesion occurred, which was the only intraoperative complication. One $5 \mathrm{~mm}$ and another $10 / 12 \mathrm{~mm}$ trocar were used in $88 \%$ of procedures while additional trocars were needed in $12 \%$. No leak of cytostatics was observed and no procedure needed to be stopped. VAS for overall difficulty of the procedure was $3 \pm 2.4$, and $3 \pm 2.9$ and $3 \pm 2.5$, respectively, for abdominal access and intraoperative staging.

Conclusions: With standardized surgical approach and dedicated safety checklist, PIPAC can be safely introduced in clinical routine with minimal learning curve.
\end{abstract}

(c) 2017 Elsevier Ltd, BASO The Association for Cancer Surgery, and the European Society of Surgical Oncology. All rights reserved.

Keywords: Intraperitoneal chemotherapy; PIPAC; Peritoneal carcinomatosis; Surgical technique; Learning curve

\section{Introduction}

Pressurized IntraPeritoneal Aerosol Chemotherapy (PIPAC) has been suggested as novel treatment modality for patients with refractory peritoneal cancer. ${ }^{1-4}$ PIPAC combines the benefits of intraperitoneal administration (increased intra-tumoral concentrations, ${ }^{5,6}$ low systemic toxicity ${ }^{4,7}$ ), pressurized vaporization (homogenous

\footnotetext{
* Corresponding author. Fax: +41 213142411.

E-mail addresses: martin.hubner@chuv.ch (M. Hübner), fabian.grass@ chuv.ch (F. Grass), hugo.teixeira-farinha@chuv.ch (H. Teixeira-Farinha), basile.pache@chuv.ch (B. Pache), patrice.mathevet@chuv.ch (P. Mathevet), demartines@chuv.ch (N. Demartines).

${ }^{1}$ Equal contribution.
}

distribution, ${ }^{2}$ deep penetration $\left.{ }^{4,5,8}\right)$ and minimal-invasive approach (repetitive application possible, low morbidity, better quality of life ${ }^{9-13}$ ). Most scientific studies on PIPAC were published by the pioneer team in Germany which elaborated also safety rules in order to minimize occupational health risks. ${ }^{1}$ Encouraging clinical results and lack of therapeutic alternatives in patients with advanced peritoneal cancer are stimuli for clinical implementation. ${ }^{9,10,12,13}$ Therefore, PIPAC is nowadays offered as treatment option in over 50 mostly European centers with about the same number of trained teams which are about to implement this novel approach in their respective institutions. This fast development entails also risks and calls for formal training, standardization and a safe implementation procedure. 
The aim of the present study was to assess practical aspects and difficulties in a non-selected consecutive series of PIPAC procedures in order to provide guidance and facilitate safe implementation of PIPAC program.

\section{Patients and methods}

PIPAC was introduced at the University Hospital of Lausanne (CHUV) in January 2015 for patients with isolated peritoneal disease that was persistent or progressive after prior standard surgical and/or medical treatment. Of note, cytoreductive surgery (CRS) and hyperthermic intraperitoneal chemotherapy (HIPEC) was preferred treatment choice for eligible patients. PIPAC was delivered in most patients as isolated treatment. Exceptionally, patients with predominating symptomatic PC and very limited systemic disease were considered for PIPAC which was then combined with concomitant systemic chemotherapy. Exclusion criteria for PIPAC were intestinal occlusion, portal vein thrombosis and medical contra-indications impeding therapeutic capnoperitoneum. Indication was confirmed by the multidisciplinary tumor board. All patients entered a prospectively maintained coded online data base (secuTrial ${ }^{\circledR}$, interactive Systems GmbH, Berlin, Germany). This analysis included all consecutive procedures until October 2016; excluded were only patients who refused to provide informed consent for participation. The study was approved by the Institutional Review Board $\left(\mathrm{N}^{\circ} 2016-00274\right)$ and online-registered under www.researchregistry.com (UIN: 1916). STROBE criteria (http://strobe-statement.org) were applied for the reporting of the study.

\section{PIPAC procedure and safety considerations (Figs. I and 2)}

Methodology and surgical approach of PIPAC procedure have been reported elsewhere $e^{4,14,15}$ and are explained in detail with the provided illustrations and photo documentation. Briefly, pneumoperitoneum was established by open placement of one $10 \mathrm{~mm}$ and one $5 \mathrm{~mm}$ balloon trocar, preferentially using existing scars from previous surgeries. Ascites was quantified, aspirated and sent for cytology. The peritoneal cancer index $(\mathrm{PCI})^{16}$ was documented and representative peritoneal nodules were biopsied. Local peritonectomy of a non-diseased area was performed to assess effects (e.g. fibrosis) of PIPAC on the healthy peritoneum.

Cytostatic solutions were prepared by clinical pharmacology on prescription by the medical oncologist and delivered to the operation room as liquid solution in securely closed plastic covers. Oxaliplatin was applied at a dose of $92 \mathrm{mg} / \mathrm{m}^{2}$ for carcinosis of colorectal origin, while Cisplatin $\left(7.5 \mathrm{mg} / \mathrm{m}^{2}\right)$ in combination with Doxorubicin $\left(1.5 \mathrm{mg} / \mathrm{m}^{2}\right)$ was used for ovarian, gastric and other malignancies. ${ }^{9,10,13}$

Liquid chemotherapy was aerosolized by use of a pressure injector (Accutron HP-D, Medtron ${ }^{\circledR}$, Saarbrücken,
Germany) and a specific nebulizer (MicroPump ${ }^{\circledR}$, CapnoPen ${ }^{\circledR}$, Reger, Villingendorf, Germany; CE-certified: class $2 \mathrm{~A}$ ) at $37{ }^{\circ} \mathrm{C}$ for $30 \mathrm{~min}$ and under standard laparoscopic pressure of $12 \mathrm{~mm} \mathrm{Hg}$. At the end of the procedure, pneumoperitoneum was evacuated in a closed aerosol evacuation system (CAWS) with two microparticle filters to capture residual molecules. PIPAC was administered repetitively ( $3 \times$ at least) at an interval of about 6 weeks.

The safety protocol included the 3 levels of containment as recommended ${ }^{1,15}$ (Fig. 2). Further, a tailored checklist containing all safety aspects (team time out, staff and procedure-related safety aspects) was systematically double-checked before administration of cytostatics. For this purpose, the original checklist from the German pioneer group was translated into French and adapted according to the experience of the first 50 PIPAC applications. The English version of the current checklist is provided as Online Appendix 1.

A standardized clinical pathway (care map) was used to support implementation and standardize and optimize perioperative care with regards to medications, nutrition, nursing and blood drawings. The initial version was adapted after careful analysis of the first 100 procedures. The current PIPAC care map is attached as Online Appendix 2.

\section{Outcome measures}

Prospectively collected data included surgical details and intraoperative findings with information on feasibility, trocar placement, biopsies, and additional procedures as detailed in Table 1. Detailed information on demographics, clinical outcomes and quality of life under PIPAC has been previously reported by our group (submitted data).

\section{Assessment of surgical difficulty}

Subjective assessment was performed by the operating surgeon $(\mathrm{MH}, \mathrm{FG}, \mathrm{HT})$ at the end of the procedure by use of a visual analogue scale (0-10: very difficult) rating abdominal access, intraoperative staging (PCI) and overall difficulty.

\section{Statistical analysis}

Categorical variables are given as frequencies with percentages and compared with chi-square test. Continuous variables are presented as mean with standard deviation (SD) or median value with range or interquartile range (IQR) as appropriate. Depending on the normality of distribution, Student's t test and Mann-Whitney U test were used. A p value $<0.05$ was considered to be statistically significant in all tests. Data analyses were generated using SPSS v20 statistical software (Chicago, IL, USA) and GraphPad Prism 7 (GraphPad Software, Inc. La Jolla, CA, USA). 
A

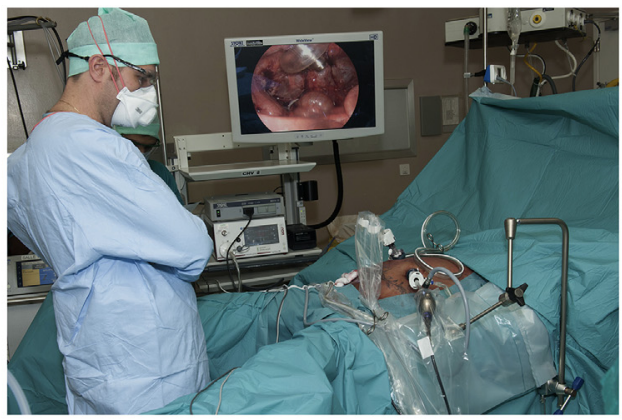

C

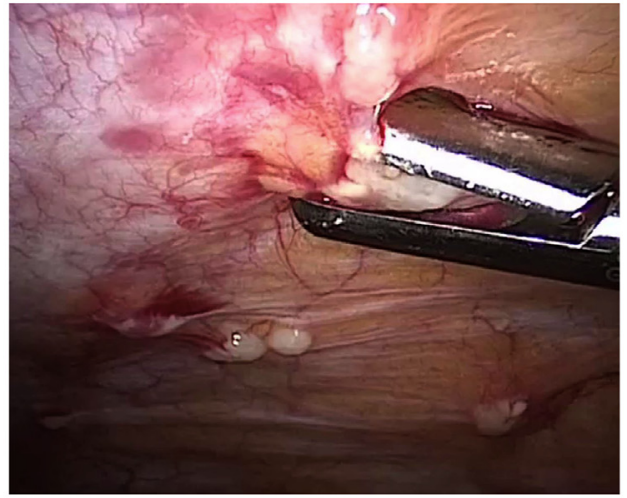

E

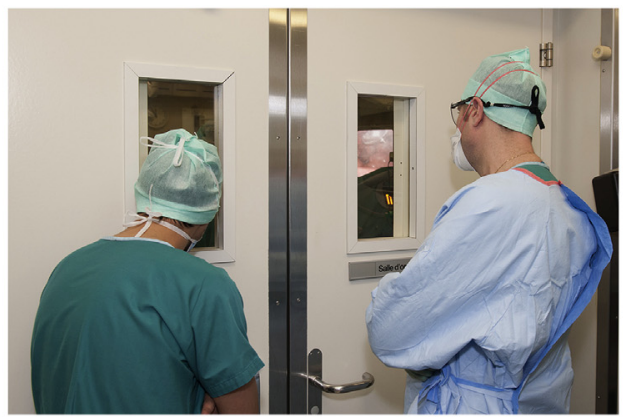

B

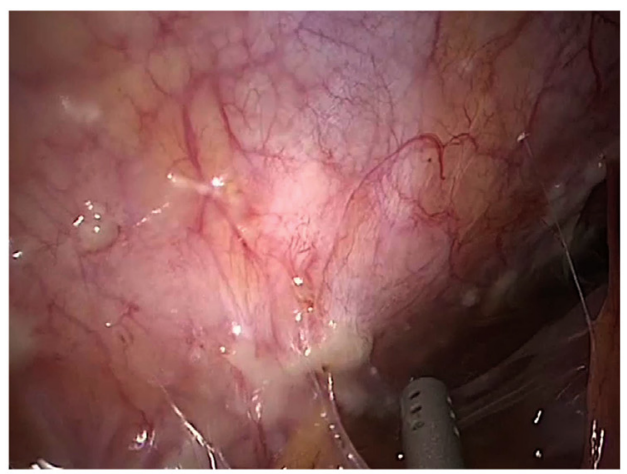

D

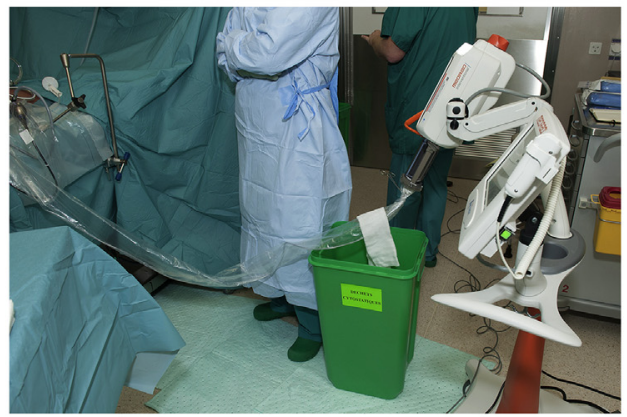

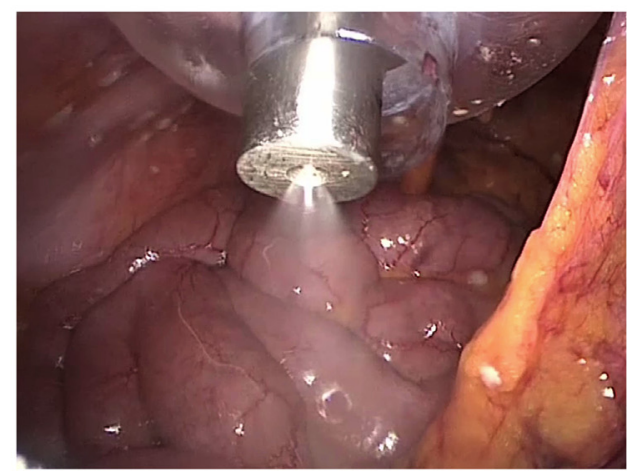

Figure 1. Standardized sequence of Pressurized IntraPeritoneal Aerosol Chemotherapy - pictorial overview. A) Positioning and trocar placement. B) Ascites drainage and exploration. C) Peritoneal biopsies and peritonectomy. D) Nebulizer, injector, evacuation system, checklist. E) Remote application. French position, scrub nurse on patient's left, screen on patient's right. Upper midline and left upper quadrant are preferred sites for Hasson trocar placement: always open access, previous incisions preferred, same incisions for consecutive PIPAC treatments, standard: one 10/12 mm, one $5 \mathrm{~mm}$ baloon trocar. Aspiration and quantification of ascites (cytology), Peritoneal cancer index (PCI), obstructive sites? Limited adhesiolysis if necessary for exploration and distribution. Representative biopsies (4+, forceps), peritonectomy (non-diseased, scissors), hemostasis (bipolar). The nebulizer is inserted in the 10/12 mm trocar with additional rubber ring. The injector is loaded with liquid cytostatic solution. Connection is performed by use of a high pressure line (+Y-connection in case of double-injection) and secured by transparent cover sheet. Connection of closed aerosol waste system (CAWS) including 2 microparticle filters and an additional valve. Correct set-up, air-tight pneumoperitoneum and respect of safety rules is double-checked by use of a standardized checklist (Online Appendix 1). Remote administration of aerosolized cytostatics with visual control of nebulization, vital signs, and proper injection on the respective screens. Nobody enters the room during administration time (nebulization +30 min). 


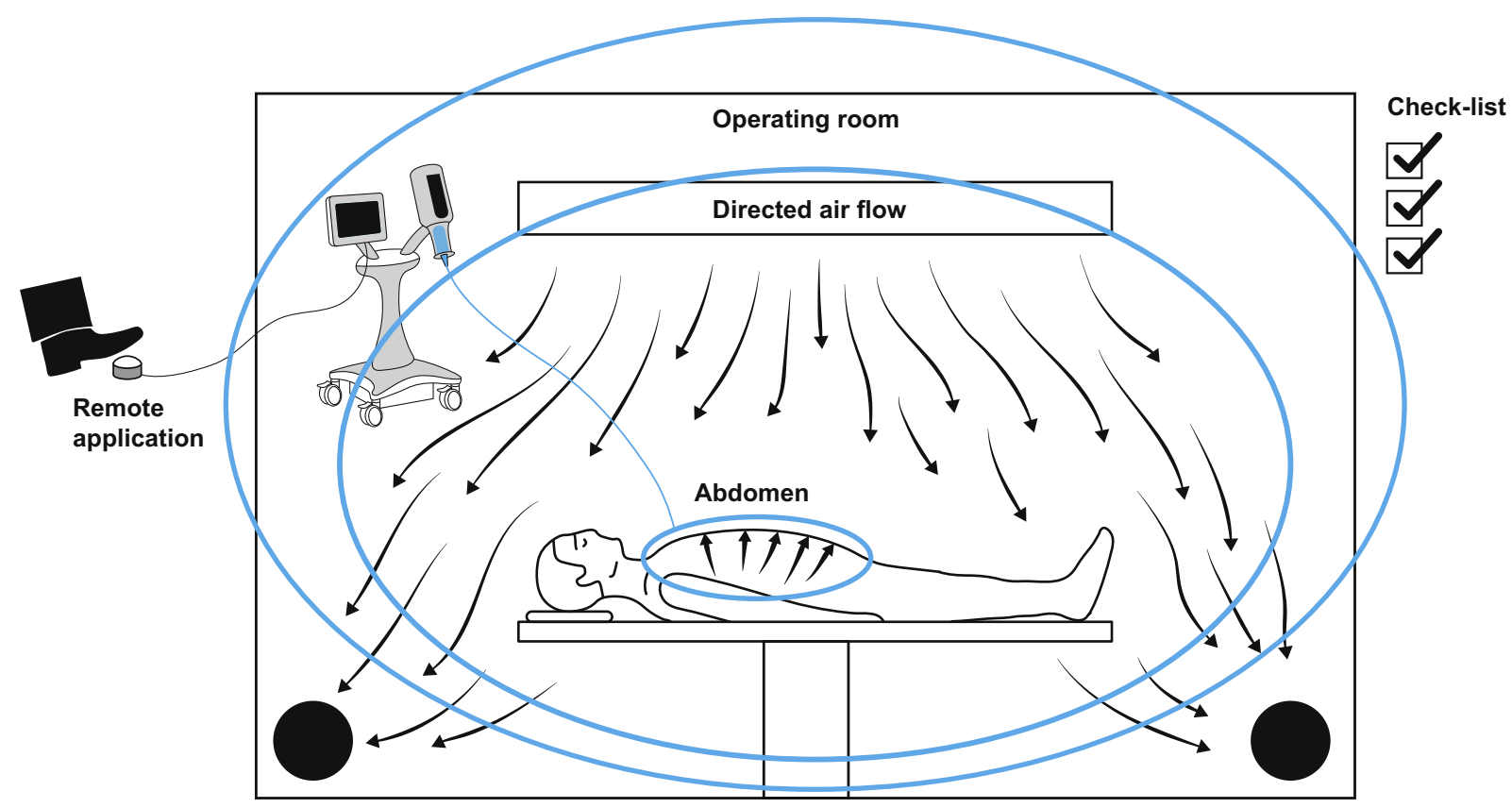

Figure 2. Pressurized IntraPeritoneal Aerosol Chemotherapy - Safety considerations. Three levels of containment ${ }^{1,15}$ assure to prevent leakage: I Air-tight pneumoperitoneum: repetitive check of the insufflator, balloon trocars. II Laminar (or directed) airflow, dilution capacity of the operation room volume. III Remote application of aersolized cytostatics from outside the room. Pressurized IntraPeritoneal Aerosol Chemotherapy can only be applied if conditions I-III have been confirmed by use of a standardized checklist (Online Appendix 1).

Table 1

Surgical details of Pressurized IntraPeritoneal Aerosol Chemotherapy.

\begin{tabular}{cllll}
\hline & $\begin{array}{l}\text { Overall } \\
(\mathrm{n}=127)\end{array}$ & $\begin{array}{l}\text { GYN } \\
(\mathrm{n}=69)\end{array}$ & $\begin{array}{l}\text { Digestive } \\
(\mathrm{n}=58)\end{array}$ & p-value \\
\hline Surgical Feasibility & & & & \\
Previous surgeries & $3(0-7)$ & $3(1-7)$ & $2(0-6)$ & 0.081 \\
$\quad$ 2 laparotomies & $44(35 \%)$ & $30(43 \%)$ & $14(24 \%)$ & 0.022 \\
Ostomy & $3(2 \%)$ & 0 & $3(5 \%)$ & 0.060 \\
Incisional hernia & $9(7 \%)$ & $6(9 \%)$ & $3(5 \%)$ & 0.440 \\
$\quad$ repaired & $6(5 \%)$ & $2(3 \%)$ & $4(7 \%)$ & 0.290 \\
No. of trocars & $2(2-5)$ & $2(2-4)$ & $2(2-5)$ & 0.856 \\
$\quad>2$ trocars & $12(9 \%)$ & $8(12 \%)$ & $7(12 \%)$ & 0.943 \\
Op. time (IQR) & $98(89-116)$ & $104(92-105)$ & $92(87-105)$ & 0.006 \\
Intra-OP findings & & & & \\
PCI (IQR) & $11(6-18)$ & $9(4-16)$ & $15(7-20)$ & 0.005 \\
Incomplete & $35(28 \%)$ & $23(33 \%)$ & $12(21 \%)$ & 0.112 \\
Ascites (mL) & $50(0-6000)$ & $50(0-5200)$ & $50(0-6000)$ & 0.199 \\
$\quad$ Cytology & $47(37 \%)$ & $26(38 \%)$ & $21(36 \%)$ & 0.863 \\
Biopsies & $126(99 \%)$ & $68(99 \%)$ & $58(100 \%)$ & 0.360 \\
$\quad$ No. of (IQR) & $3(3-4)$ & $3(2-4)$ & $4(3-4)$ & 0.460 \\
Peritonectomy & $115(91 \%)$ & $61(88 \%)$ & $54(93 \%)$ & 0.367 \\
Adhesiolysis & $21(16 \%)$ & $13(18 \%)$ & $8(15 \%)$ & 0.445 \\
Additional surgery & $21(17 \%)$ & $10(14 \%)$ & $11(19 \%)$ & 0.499 \\
\hline
\end{tabular}

Median (range, unless specified) and N (\%) as appropriate.

Statistical significance $(\mathrm{p}<0.05)$ is highlighted in bold.

PCI: peritoneal cancer index.

Additional surgery included hernia repair $(n=6)$, resection of trocar metastases $(\mathrm{n}=8)$, omentectomy $(\mathrm{n}=2)$, correction of hypertrophic scars $(\mathrm{n}=2)$, wedge resection of liver metastasis $(\mathrm{n}=1)$, inguinal hernia repair $(\mathrm{n}=1)$ and intraoperative esophagogastroduodenoscopy $(\mathrm{n}=1)$.

\section{Results}

From January 2015 until October 2016, 131 PIPAC procedures were scheduled for 60 patients. Primary non-access rate was $2 / 60(3.3 \%)$ patients and secondary non-access rate was 1/69 PIPAC procedures (1.4\%), giving an overall nonaccess rate of 3/131 (2\%). In addition, one PIPAC administration was deferred due to incidental small bowel injury. No patient refused consent for utilization of clinical data, and the present analysis included therefore all consecutive 127 procedures. Patient recruitment was stable over time with 5-6 procedures performed per month (Fig. 3). Overall, there were 9 postoperative complications (7\%) including one mortality due to cardiac arrhythmia 4 days after the 3rd PIPAC application. No causative relation with PIPAC treatment was identified by autopsy. Eight minor complications were urinary retention $(3 \times)$, wound complications $(2 \times)$, postoperative ileus, constipation and transitory neutropenia $(1 \times$ each). Median postoperative hospital stay was 3 days $(2-15)$ for PIPAC1-20, 3 days $(2-10)$ for PIPAC2150 , and 2 days (1-7) for PIPAC51-127 $(p=0.015)$.

\section{Surgical details}

Surgical feasibility and intraoperative findings for 69 gynecological and 58 digestive PIPAC procedures are detailed in Table 1. Of note, median number of prior surgeries was 3 $(0-4)$ with 44 patients $(35 \%)$ having $\geq 2$ prior laparotomies. Ostomy and incisional hernia were present in 3 


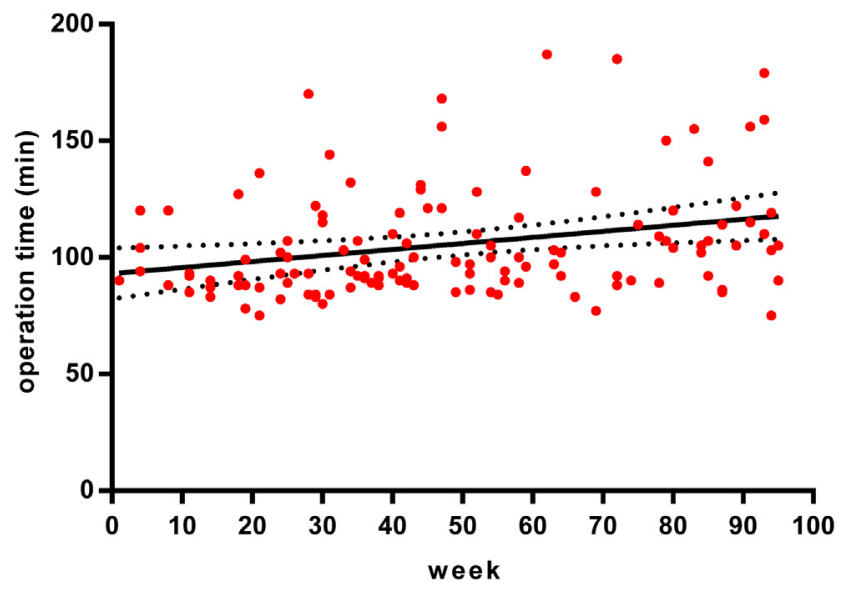

Figure 3. Pressurized IntraPeritoneal Aerosol Chemotherapy (PIPAC) Recruitment and operation times over time. Patient accrual remained stable over time, while operation time increased $(\mathrm{p}=0.025)$.

(2\%) and $9(7 \%)$ cases, respectively. Synchronous hernia repair was performed in 6 out of 9 cases $(67 \%)$ during PIPAC\#1 $(\mathrm{n}=2)$, PIPAC\#2 $(\mathrm{n}=3)$ or PIPAC\#3 $(\mathrm{n}=1)$, respectively. Hernia sac was used for Hasson trocar placement in 5 cases (4\%). Hernia sac was resected in one patient prior to PIPAC with placement of Gelport ${ }^{\circledR}$ to assure air-tight pneumoperitoneum. Synthetic mesh was used in 2 patients, while suture repair was performed in the remaining 4 patients. Adhesiolysis was performed in 21 patients $(16 \%)$. Additional procedures are detailed in Table 1 . The minimum number of two trocars was utilized in 115 procedures $(91 \%)$. The preferred sites for trocar placement were upper midline, left upper quadrant and left flank as depicted in Fig. 4A. Median operation time was overall $98 \mathrm{~min}$ (IQR 89-117) with $91 \mathrm{~min}$ (IQR 87-103), $93 \mathrm{~min}$ (IQR 88-107), and $103 \mathrm{~min}$ (IQR 91-121) for PIPAC 1-20, PIPAC 21-50 and PIPAC 51-127, respectively (Fig. 3).

Intraoperative findings and sequence according to the standardized procedure (Fig. 1) are displayed in Table 1. PCI was documented in all cases, but laparoscopic staging was incomplete in 35 procedures $(28 \%)$. Non-accessible areas due to dense adhesions or extensive peritoneal disease are displayed in Fig. 4B. A median of 3 (IQR 3-4) biopsies was retrieved; biopsy sites are depicted in Fig. 4C.

Except for the nebulizer, no specific material was necessary. Biopsy forceps and monopolar scissors were used for peritoneal biopsies and peritonectomy, respectively, while bipolar forceps were used to achieve hemostasis.

\section{Technical problems and safety}

No intervention had to be abandoned for technical or safety issues. Safety checklist (Online Appendix 1) was followed in all patients, and omissions could hence be avoided in 11 procedures $(8.6 \%)$. These were not-installed CAWS $(\mathrm{n}=2)$, open CAWS $(\mathrm{n}=3)$, missing transparent sheet
( $\mathrm{n}=1$ ), missing cytostatic waste bin underneath the injector head $(\mathrm{n}=2)$ and missing protective eye wear (circulating nurse, $\mathrm{n}=5$ ). Checklist was successfully completed in all cases before administration of cytostatics.

Fourteen minor events occurred during aerosolization (11\%). Y connection of the high pressure line was defective and had to be replaced in 5 procedures $(3.9 \%)$. The resulting leak was entirely contained by the transparent cover sheet around the pressure line in all instances. No single leak was recorded when performing single-head injection. Pressure limitation (>200PSI) was attained in 9 cases (7.1\%) leading to an automatic safety stop of injection. Administration could be completed after a short pause $(<1 \mathrm{~min})$ in all cases respecting the defined pressure limits. During one procedure, the injector was non-functional because the OR team had omitted to charge overnight. The procedure could be continued after $15 \mathrm{~min}$ of charging time.

\section{Surgical difficulty}

Procedural difficulty showed large variations (Fig. 5). Subjective assessment (VAS) of abdominal access and reliability of laparoscopic staging was a median of 3 (IQR $1-5$ ) and 3 (IQR 1-6), respectively. Overall difficulty of the procedure was rated at 2 (IQR $1-5)$.

\section{Discussion}

Pressurized IntraPeritoneal Aerosol Chemotherapy (PIPAC) was implemented as a highly standardized procedure with a strict safety protocol. Formal training and respect of the available recommendations allowed for safe implementation with no learning curve, and reproducible procedural results.

PIPAC is a new treatment modality that was available until 2014 only in the pioneer center in Germany. Access to the technology (nebulizer) is allowed only to centers that followed formal training (certification course) on background, technical aspects and safety concept. This report described results, problems and practical aspects of a consecutive series of procedures during implementation of a PIPAC program. Abdominal access rate and repeatability was high as it was reported from the German pioneer group and elsewhere. ${ }^{12,13}$ Operation time showed little variation and was similar to previous reports. ${ }^{4,12}$ Interestingly, no learning curve was observed as operation times did not decrease over time. This could be attributed to the fact that PIPAC procedure was highly standardized as outlined in the accompanying material (Figs. 1 and 2). Standardization has been shown in various fields to decrease learning curve and to improve performance. ${ }^{17,18}$ In analogy to aviation, a strict safety protocol was developed by the German pioneer group. ${ }^{1}$ Safety checklists have been shown to decrease avoidable errors. This was the case in $8.6 \%$ of the PIPAC procedures in this report. Although these were 
A

Trocar placement

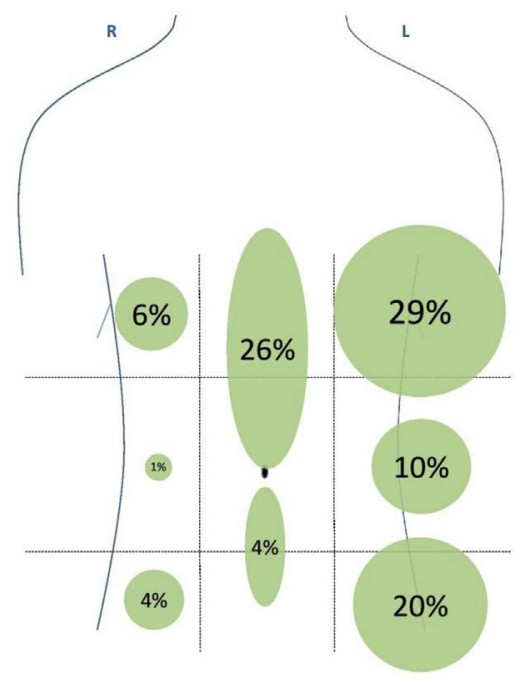

C

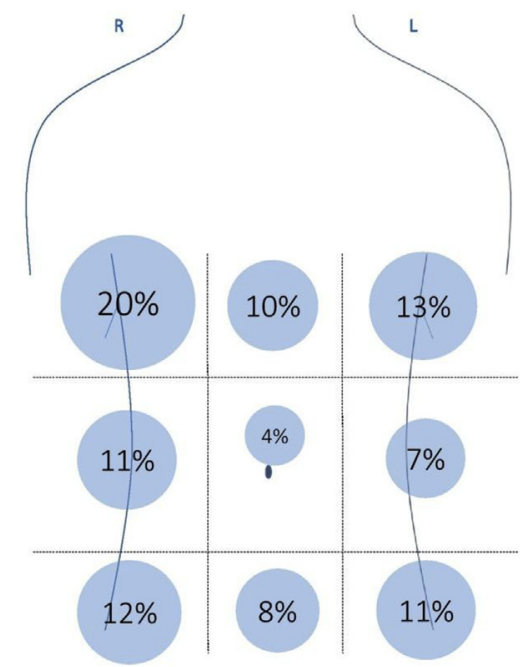

B Inaccessible areas

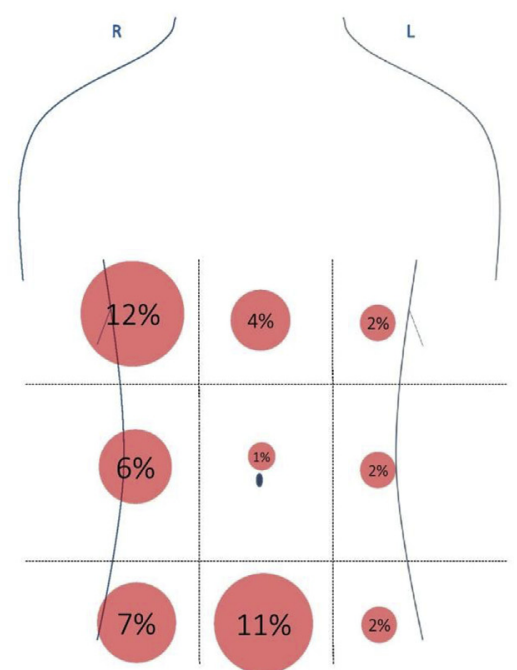

Figure 4. Pressurized IntraPeritoneal Aerosol Chemotherapy - Trocar placement, inaccessible areas and biopsy sites. A) Preferred sites of trocar placement $(\mathrm{n}=268)$ in \%. Left upper quadrant $(29 \% ; \mathrm{n}=78)$ and upper midline $(26 \% ; \mathrm{n}=68)$ were preferred sites for open placement of Hasson trocar. Previous incisions were preferred to reduce potential trocar metastasis. B) Inaccessible areas for exploration/staging due to severe adhesions or dense peritoneal implants resulting in incomplete staging in $\%$ of all procedures $(n=127)$. C) Biopsy sites $(n=431)(\geq 4$ biopsies/procedure if feasible $)$ in $\%$.

all minor omissions, it underlines the usefulness of a checklist that takes an additional time of only 1-2 min (Online Appendix 1). The additional value of a checklist and intraoperative events have not been reported by other PIPAC groups. Therefore, we cannot provide comparative data to the intraoperative event rate of $11 \%$ reported in the present study. These events occurred despite diligent performance and under strict respect of the approved material and treatment protocol. Centers starting a PIPAC program should therefore be aware of these incidents that are unlikely to entail any consequences for patients and staff as long as safety guidelines are meticulously followed.
PIPAC is not a technically demanding procedure but abdominal access in multioperated patients may be challenging. Due to the current indications, the large majority of PIPAC patients had prior abdominal surgery. Patients in this study had a median of 3 prior abdominal surgeries and $35 \%$ had 2 or more laparotomies in the past. Laparoscopic access was still feasible in all cases except 3 with prior cytoreductive surgery and HIPEC. Adhesions are reported in nearly all patients with prior surgery at various degrees. ${ }^{19}$ However, adhesiolysis should be limited due the potentially fatal consequences of serosal tears. Main indication is therefore insufficient access to the different 

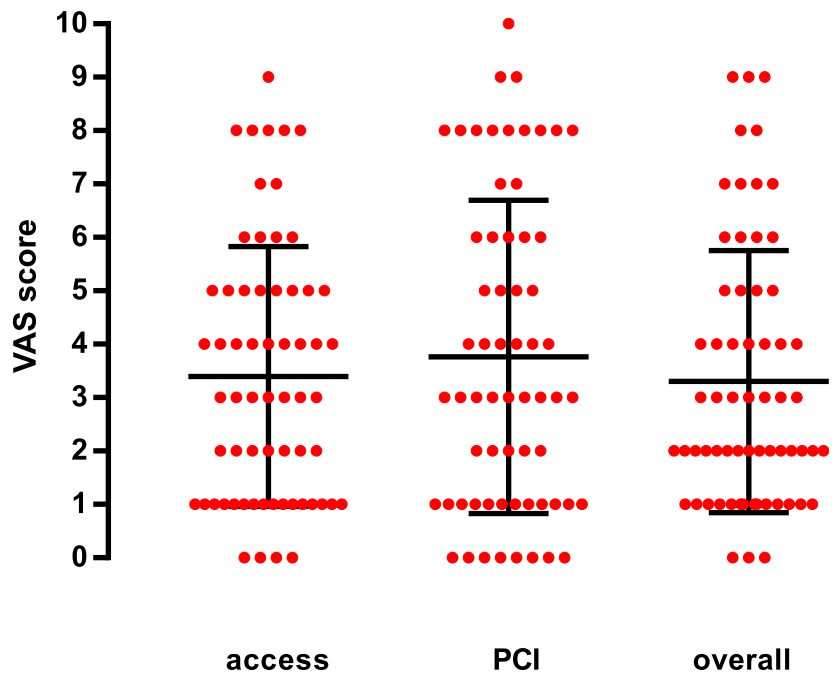

Figure 5. Pressurized IntraPeritoneal Aerosol Chemotherapy - surgical difficulty. Subjective assessment of overall difficulty, access, and staging (PCI - peritoneal cancer index) by use of a visual analogue scale (VAS: 0-10: extremely difficult). Displayed are individual values per procedure and median (IQR).

abdominal areas limiting staging but also free distribution of cytostatics. In this present cohort, limited adhesiolysis was necessary in $16 \%$ of procedures, mostly during the first PIPAC procedure. PIPAC by itself does not appear to cause adhesions, and trocar sites of PIPAC\#1 can therefore usually be used also for the following treatments. Existing incisions should preferably be used for patients with peritoneal metastases in order to limit sites of potential tumor seeding. On the other hand, intestinal structures are frequently adherent to a midline incision, and alternative access may be required. Trocars should be limited in order to decrease the risk for trocar metastases and in order to diminish the potential for air leaks during administration of cytostatic agents. In this series, PIPAC procedures were performed with the minimal number of one $5 \mathrm{~mm}$ and one $10 / 12 \mathrm{~mm}$ trocar in $91 \%$ of cases. Preferred trocar sites were the upper midline and left upper quadrant as displayed in Fig. 4A. With this strategy, only one single small bowel lesion occurred in 127 reported procedures.

This study reports a single-center experience that cannot uncritically be generalized to other settings. However, the present results are in line with reports from the pioneer center indicating reproducibility of this standardized procedure. The current standards are mostly empirical but appear to be useful in clinical practice. Changes should be performed only after careful assessment of risks and benefits.

In conclusion, formal training, adherence to the standardized procedure and established safety protocol allow for safe implementation of PIPAC with no learning curve. The available recommendations and the information material provided in this report can help to prevent avoidable errors and to establish uniform treatment standards.

\section{Conflict of interest statement}

No conflict of interest to declare for all authors.

\section{Sources of support}

None.

\section{Acknowledgement}

No funding sources to declare.

\section{Appendix A. Supplementary data}

Supplementary data related to this article can be found at http://dx.doi.org/10.1016/j.ejso.2017.03.019.

\section{References}

1. Solass W, Giger-Pabst U, Zieren J, Reymond MA. Pressurized Intraperitoneal Aerosol Chemotherapy (PIPAC): occupational health and safety aspects. Ann Surg Oncol 2013;20(11):3504-11.

2. Solass W, Herbette A, Schwarz T, et al. Therapeutic approach of human peritoneal carcinomatosis with Dbait in combination with capnoperitoneum: proof of concept. Surg Endosc 2012;26(3): 847-52.

3. Solass W, Hetzel A, Nadiradze G, Sagynaliev E, Reymond MA. Description of a novel approach for intraperitoneal drug delivery and the related device. Surg Endosc 2012;26(7):1849-55.

4. Solass W, Kerb R, Murdter T, et al. Intraperitoneal chemotherapy of peritoneal carcinomatosis using pressurized aerosol as an alternative to liquid solution: first evidence for efficacy. Ann Surg Oncol 2014; 21(2):553-9.

5. Esquis P, Consolo D, Magnin G, et al. High intra-abdominal pressure enhances the penetration and antitumor effect of intraperitoneal cisplatin on experimental peritoneal carcinomatosis. Ann Surg 2006; 244(1):106-12.

6. Facy O, Al Samman S, Magnin G, et al. High pressure enhances the effect of hyperthermia in intraperitoneal chemotherapy with oxaliplatin: an experimental study. Ann Surg 2012;256(6):1084-8.

7. Blanco A, Giger-Pabst U, Solass W, Zieren J, Reymond MA. Renal and hepatic toxicities after Pressurized Intraperitoneal Aerosol Chemotherapy (PIPAC). Ann Surg Oncol 2013;20(7):2311-6.

8. Minchinton AI, Tannock IF. Drug penetration in solid tumours. Nat Rev Cancer 2006;6(8):583-92.

9. Demtroder C, Solass W, Zieren J, Strumberg D, Giger-Pabst U, Reymond MA. Pressurized Intraperitoneal Aerosol Chemotherapy (PIPAC) with oxaliplatin in colorectal peritoneal metastasis. Colorectal Dis Off J Assoc Coloproctol G. B Irel 2015;18(4):364-71.

10. Nadiradze G, Giger-Pabst U, Zieren J, Strumberg D, Solass W, Reymond MA. Pressurized Intraperitoneal Aerosol Chemotherapy (PIPAC) with low-dose cisplatin and doxorubicin in gastric peritoneal metastasis. J Gastrointest Surg Off J Soc Surg Aliment Tract 2015; 20(2):367-73.

11. Odendahl K, Solass W, Demtroder C, et al. Quality of life of patients with end-stage peritoneal metastasis treated with Pressurized IntraPeritoneal Aerosol Chemotherapy (PIPAC). Eur J Surg Oncol J Eur Soc Surg Oncol Br Assoc Surg Oncol 2015;41(10):1379-85.

12. Robella M, Vaira M, De Simone M. Safety and feasibility of Pressurized Intraperitoneal Aerosol Chemotherapy (PIPAC) associated with 
systemic chemotherapy: an innovative approach to treat peritoneal carcinomatosis. World J Surg Oncol 2016;14:128.

13. Tempfer CB, Winnekendonk G, Solass W, et al. Pressurized Intraperitoneal Aerosol Chemotherapy in women with recurrent ovarian cancer: a phase 2 study. Gynecol Oncol 2015;137(2):223-8.

14. Hubner M, Teixeira H, Boussaha T, Cachemaille M, Lehmann K, Demartines N. PIPAC-Pressurized Intraperitoneal Aerosol Chemotherapy. A novel treatment for peritoneal carcinomatosis. Rev Med Suisse 2015;11(479):1325-30.

15. Reymond ME, Solass W. PIPAC - Pressurized Intraperitoneal Aerosol Chemotherapy - cancer under pressure. Berlin/Boston: Walter de Gruyter; 2014.
16. Portilla AG, Shigeki K, Dario B, Marcello D. The intraoperative staging systems in the management of peritoneal surface malignancy. J Surg Oncol 2008;98(4):228-31.

17. Luglio G, De Palma GD, Tarquini R, et al. Laparoscopic colorectal surgery in learning curve: role of implementation of a standardized technique and recovery protocol. A cohort study. Ann Med Surg 2015;4(2):89-94.

18. Loftus T, Dahl D, OH B, et al. Implementing a standardized safe surgery program reduces serious reportable events. J Am Coll Surg 2015; 220(1):12-7. e13.

19. Strik C, Stommel MW, Schipper LJ, van Goor H, Ten Broek RP. Longterm impact of adhesions on bowel obstruction. Surgery 2016;159(5): 1351-9. 